\title{
Chain-link-fence structures produced in a plane jet
}

\author{
Jun Sakakibara and Tomokuni Anzai \\ Institute of Engineering Mechanics and Systems, University of Tsukuba, Tsukuba 305-8573, Japan
}

(Received 9 January 2001; accepted 15 March 2001)

\begin{abstract}
Vortical structures of the chain-link-fence type have been experimentally produced in a plane jet. The jet was excited by temporal periodic disturbances with spanwise phase variations added in the initial shear layer. Chain-link-fence-like structures, which significantly differed from ordinary vortices such as "roller and rib" structures, are observed experimentally by stereoscopic particle image velocimetry when the temporal phase difference of disturbances with the spanwise direction is $180^{\circ}$. Some of the original vortices remain downstream as stretched lambda-type vortices, although the destruction of the orderly vortices into complex turbulence was significant. (C) 2001 American Institute of Physics. [DOI: 10.1063/1.1370391]
\end{abstract}

Manipulation of free shear flow is often accomplished by adding artificial disturbances to the initial shear layer to excite the primary (Kelvin-Helmholz) (K-H) instability, with the disturbance actually triggering the first roll-up of the shear layer. ${ }^{1,2}$ In addition to K-H instability, secondary instability such as translative instability, ${ }^{3}$ which induces a sinusoidal distortion of the spanwise rollers, or braid instability, which generates streamwise vortices such as rib structures, ${ }^{4}$ can be triggered by adding spanwise nonuniform disturbances. ${ }^{5,6}$ Lasheras and $\mathrm{Choi}^{5}$ used a fluted splitter plate in their plane mixing layer apparatus to dislocate the origin of the mixing layer periodically in a spanwise direction. Their method was applied to add spanwise periodic streamwise vorticity in the initial stage of the shear layer and form equally spaced rib vortices that connected braids of successive rollers. While their method was passive, Nygaard and Glezer $^{6}$ established active control of the mixing layer. They placed a linear array of individually controlled surface heaters on the splitter plate, and time harmonic wave trains were introduced into the heaters. If the waves to the adjacent heater segments were out of phase, the spanwise roller and rib vortices exhibited chain-link-fence-like structures.

In the present letter, we observed a structure similar to Nygaard and Glezer's, but in a forced plane jet. The initial shear layer of the jet was excited by an array of suction/ blowing miniature jets exiting normal to the streamwise direction of the plane jet. The visualization of the structures was accomplished by a time-resolving stereoscopic particle image velocimetry, and a quasi-three-dimensional vorticity field could be visualized by assuming Taylor's frozen hypothesis.

The experiment was conducted in planar water jet apparatus. Water in a reservoir was introduced into a settling chamber having a flow straightener and a mesh. The flow then passed through a smooth contraction nozzle and issued upward into a rectangular Plexiglas tank $(640 \times 680$ $\times 300 \mathrm{~mm}^{3}$, in height, width, and span) forming a laminar plane jet with rectangular velocity profile. The nozzle, having a width $B=30 \mathrm{~mm}$ and an aspect ratio equal to 10 at the exit, was designed to add a suction/blowing-type distur- bance: it has a row of 60 rectangular slots at the right side $(x, y)=(-0.33 B, 0.5 B)$ and the same at the left side $(-0.33 B,-0.5 B)$ aligned in a spanwise direction as illustrated in Fig. 1, which also indicates the coordinate system. The cross section of the slot was $1 \times 4 \mathrm{~mm}^{2}$ in height and span, with interval between center of each slot being $\Delta=B / 6$. Each slot lead via plastic tubes to a single manifold, which combined seven or eight other tubes coming from every eighth slot into one. The manifolds were then connected to 1 of 16 glass syringes, each with a capacity of $2 \mathrm{ml}$. The pistons of the syringes were driven by PC-controlled servo motors (Futaba, S3003). Thus, on both sides were 16 sets of syringes and servomotors, each of which could produce a suction or blowing miniature slot jet. The motion of the piston in the syringe connected to a slot at spanwise location $z$ was determined by a control signal sent to the servomotor expressed as

$$
\begin{aligned}
& X_{S}=A \operatorname{Sqr}[2 \pi f t+\Psi(z)+\psi], \\
& \Psi(z)=\frac{\phi}{2} \operatorname{Sqr}\left(\frac{2 \pi z}{\lambda_{z}}\right),
\end{aligned}
$$

where $X_{S}$ is the desired position of the piston head measured from the end of the syringe, $A$ is the amplitude of the piston oscillation, Sqr is a square wave with a unit amplitude and $50 \%$ duty cycle with phasing like the sine function, $\phi$ is the amplitude of the phase variation, and $\psi$ is the phase lag between signals for the shear layer at the left and right sides. The blowing (suction) slot jet was produced at the rising (falling) edge of the signal. Typical slot jet velocity $v_{j}$ estimated from a measured position of the piston is shown in Fig. 2. Note that the actual slot jet velocity might not be identical to this figure because the plastic tubes between syringes and slots could be slightly deformed by the oscillating pressure inside.

The spanwise wave length $\lambda_{z}$ of the phase variation was set at $\lambda_{z}=1.33 B$. The jet Reynolds number was set at $\operatorname{Re}=B U_{0} / \nu=2300$, where the jet exit velocity was $U_{0}=80 \mathrm{~mm} / \mathrm{s}$. The jet Strauhal number was set at $\mathrm{St}$ $=f B / U_{0}=0.45$ where the frequency $f(=1 / T)$ was chosen 


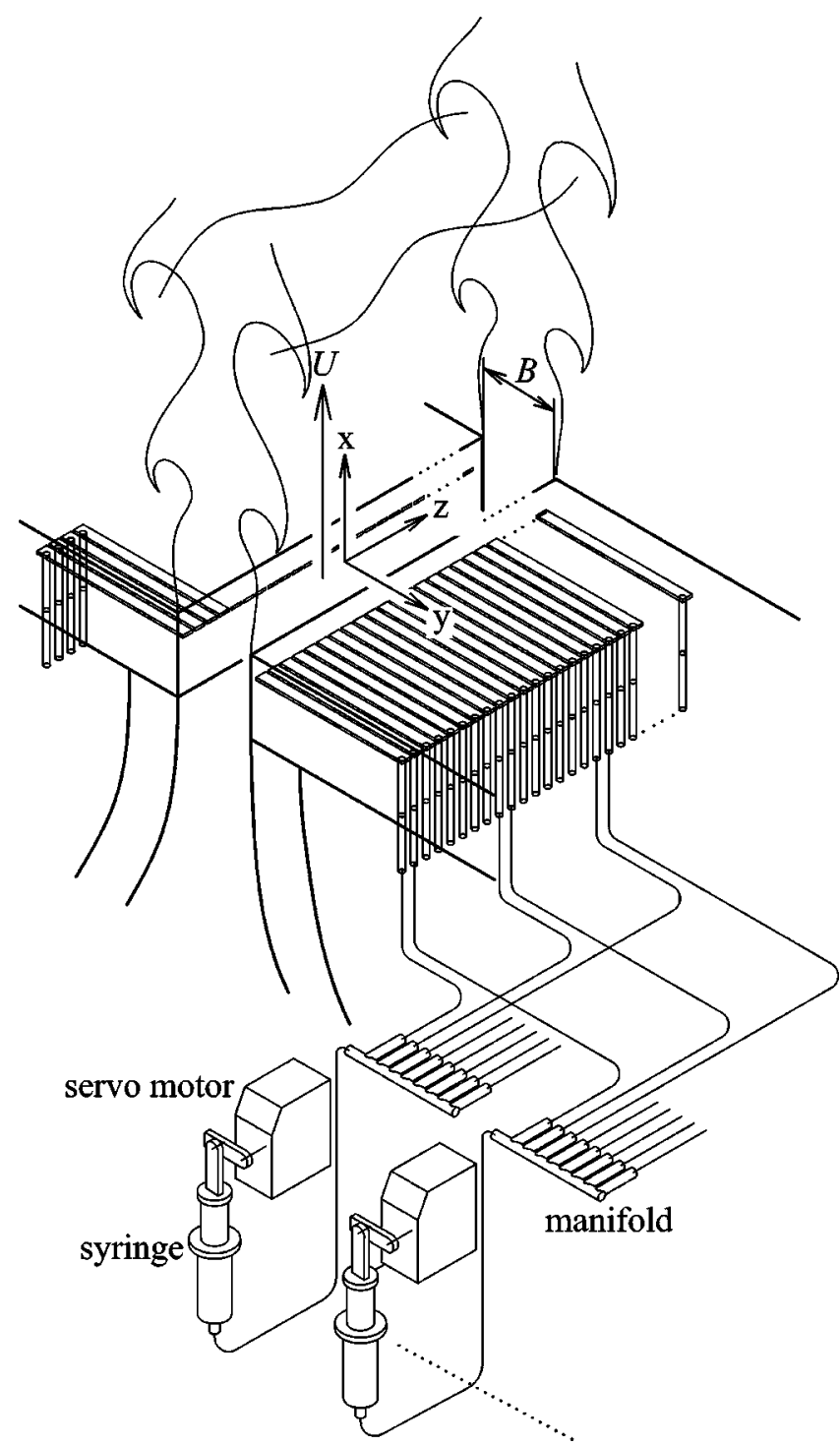

FIG. 1. Details of the nozzle exit. Rows of rectangular slots lead into syringes through plastic tubes and manifolds. Eight sets of syringes, servo motors, and manifolds are used on each side to realize variable spanwise wave lengths.

to be the most preferred frequency. The corresponding streamwise wave length was $\lambda_{x}=U_{0} / 2 f=1.11 B$ assuming a convection velocity equal to $U_{0} / 2$. The phase lag between shear layers $\psi$ was set at 0 , which created disturbance in both the shear layers in-phase.

Velocity measurement was achieved by the stereoscopic PIV technique, which is capable of resolving time-dependent three-component velocity in a two-dimensional plane. Although the original idea of our custom-made stereoscopic PIV system was based on Ref. 7, we used third-order polynomials as mapping functions between the physical coordinate and the image coordinate, which were obtained by a calibration procedure similar to that described in Ref. 8.

A laser light sheet produced by a Nd-YAG laser (New Wave Research, MiniLase) illuminated a plane normal to the streamwise direction at $x / B=2$ or 4 , and two CCD cameras (Kodak, ES1.0) were placed to view the same region of interest in the light sheet plane. The angle between the axes of

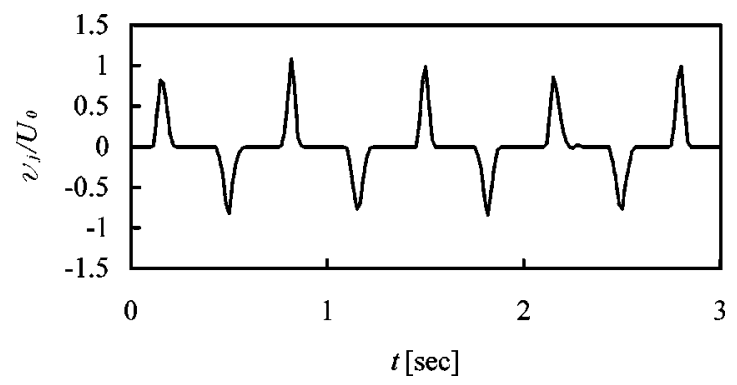

FIG. 2. Temporal variation of the slot jet velocity estimated from the measured position of the piston in the syringes. Peak velocity of the impulsive slot jet $v_{j}$ is close to the main flow velocity $U_{0}$, and its duration was approximately $50 \mathrm{~ms}$.

the two cameras was about $90^{\circ}$, and lenses were mounted to satisfy the Scheimpflug condition. The images captured by the CCD cameras were stored into the host memory of a PC through image grabbers (Imaging Technology, IC/PCI) with a frame rate of $30 \mathrm{fps}$. Our PIV system could typically capture 150 time-dependent successive images for both cameras. Since an instantaneous velocity field could be measured based on the double images, 75 instantaneous threecomponents velocity maps with $\frac{1}{15} \mathrm{~s}$ intervals were obtained for every run of experiments.

Although our stereo PIV system could resolve velocity distribution only on a two-dimensional plane, the axis normal to the plane was defined as $x^{*}=-t U_{C}$ based on the Taylor's frozen hypothesis. Here $U_{C}$ is the convection velocity of the structures, approximated as $U_{C}=0.5 U_{C L}$, and $U_{C L}$ is a centerline mean velocity of the jet at given $x$ location. Thus the vorticity vector is given as

$\omega=\left(\frac{\partial w}{\partial y}-\frac{\partial v}{\partial z}\right) \mathbf{i}+\left(\frac{\partial u}{\partial z}-\frac{\partial w}{\partial t} \frac{\partial t}{\partial x^{*}}\right) \mathbf{j}+\left(\frac{\partial v}{\partial t} \frac{\partial t}{\partial x^{*}}-\frac{\partial u}{\partial y}\right) \mathbf{k}$,

where $\mathbf{i}, \mathbf{j}, \mathbf{k}$ are unit vectors parallel to $x, y$, and $z$ axes, respectively. The results shown in Fig. 3 represent surfaces of constant vorticity magnitude $|\boldsymbol{\omega}|=2.5 U_{0} / B$ measured in the $y-z$ plane at $x / B=2$. The mean flow direction is provided by an allow in Fig. 3(a). The structure for $\phi=0$ in Fig. 3(a) clearly depicts spanwise rollers in each shear layer of the jet. Since the disturbances added to each shear layer were in phase $(\psi=0)$, the rollers are symmetrical in respect to the center plane of the jet. In each shear layer, five rollers are displayed in the figure. Here every two rollers are close to each other and are likely to exhibit vortex pairing further downstream. Since the disturbance was two-dimensional and had no spanwise phase variation, the secondary instability was not triggered explicitly, unlike the following case of $\phi \neq 0$. Thus, neither the sinusoidal distortion of the rollers nor the streamwise rib vortices due to the secondary instability are obvious in this stage.

In the case of $\phi=3 \pi / 4$, the sinusoidal distortion of the rollers is clear, as shown in Fig. 3(b). The streamwise rib vortices, which are similar to the horns in this picture, are also formed between the adjacent roller pairs.

Figure 3(c), where $\phi=\pi$, exhibits more pronounced features of the structures; it consists of sinusoidal vortices arranged regularly but $180^{\circ}$ out of phase between the adjacent 

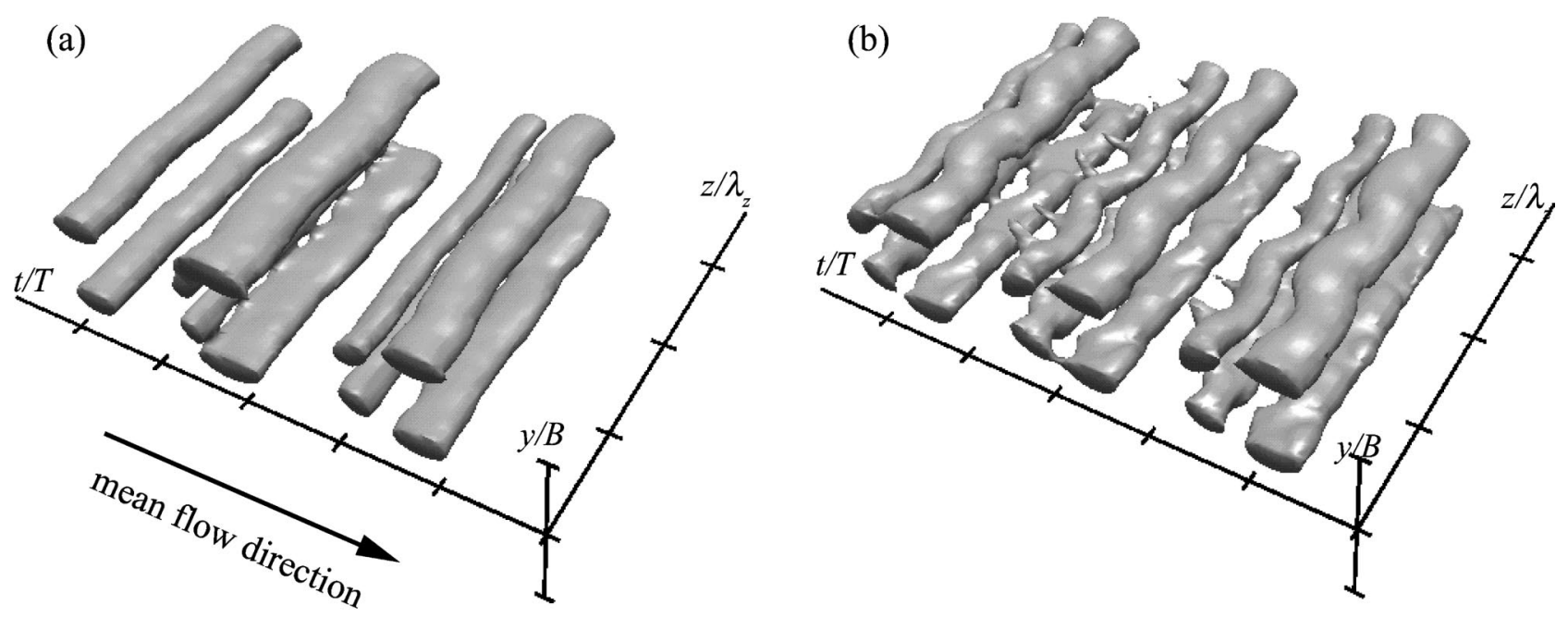

(c)

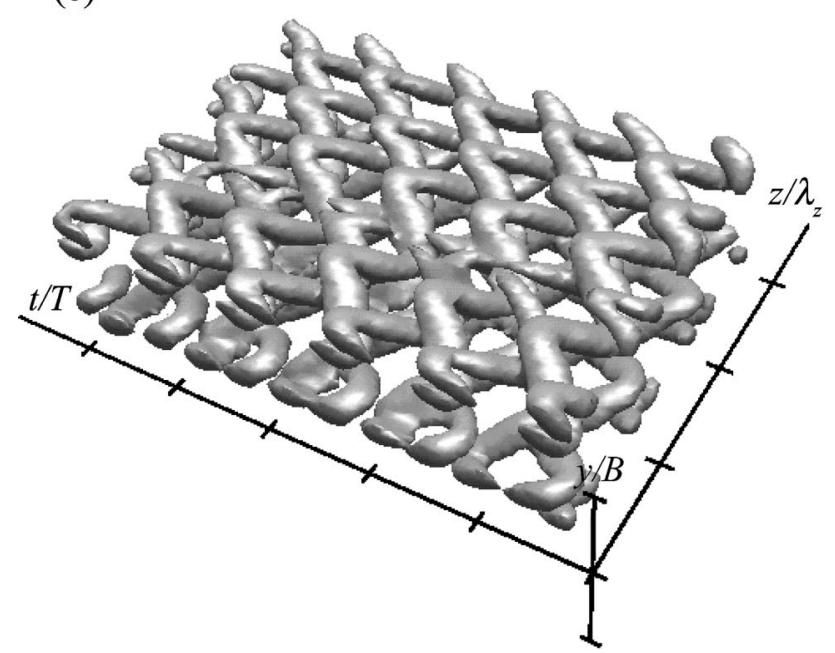

FIG. 3. Surfaces of constant vorticity magnitude threshold at $|\omega|=2.5 U_{0} / B$ calculated from the time-dependent PIV data measured at $x / B=2 ;$ (a) $\phi=0$, (b) $\phi=3 \pi / 4$, (c) $\phi=\pi$. Tic intervals are respectively $1.0 T, 1.0 B$, and $1.0 \lambda_{z}$ in $t-, y$-, and $z$-directions.

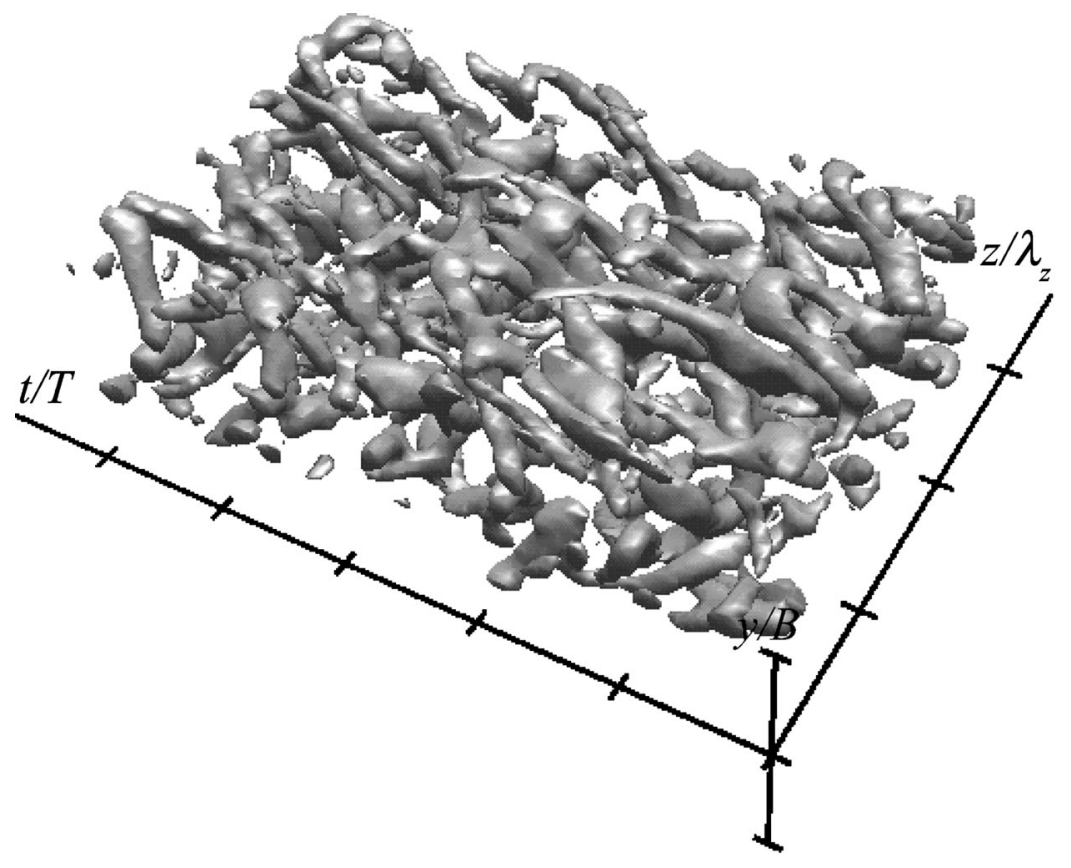

FIG. 4. Surfaces of constant vorticity magnitude threshold at $|\boldsymbol{\omega}|=2.5 U_{0} / B$ calculated from the timedependent PIV data measured at $x / B=4$ for the case $\phi=\pi$. Tic intervals are the same as those of Fig. 3. 
one in the streamwise direction. This forms the "chain-linkfence-like" structure described in Ref. 9. The downstream head of the sinusoidal vortex dislocates toward the center plane of the jet, and the tail of neighboring vortex covers it from the outside. These are most likely to be the lambda-type vortices repeated in the spanwise and streamwise directions. Similar structures have been observed experimentally in plane mixing layers by Nygaard and Glezer, ${ }^{6}$ and simulated numerically by Collis et al. ${ }^{9}$ The present result is the first observation of such structures in the plane jet.

Nygaard and Glezer ${ }^{6}$ observed that the chain-link-fence pattern was not formed if the wave angle is greater than a cutoff angle of approximately $45^{\circ}$. By Collis et al., ${ }^{9}$ on the other hand, the cutoff angle of the experiments by Nygaard and Glezer ${ }^{6}$ was estimated as $56^{\circ}$ according to linear stability analysis. Since the wave angle of the present case $\left(40^{\circ}\right)$ was relatively smaller than the above criterion, the chain-linkfence pattern was expected to appear. Note that we did not observe the chain-link-fence pattern in the case of $\lambda_{z}=0.67 B$, which is half the wavelength above.

Further downstream, $x / B=4$, the chain-link-fence structures were broken into turbulence and exhibited more a complicated vorticity field, as shown in Fig. 4. However, the lambda-type vortices were still observed in some part of the flow. They were further stretched toward the streamwise and transverse direction.

\section{ACKNOWLEDGMENT}

This work was supported by a grant-in-aid for scientific research by the Ministry of Education of Japan under Grant No. 10750140.

${ }^{1}$ C. M. Ho and L. S. Huang, "Subharmonics and vortex merging in mixing layers,' J. Fluid Mech. 119, 443 (1982).

${ }^{2}$ D. Oster and I. Wygnanski, "The forced mixing layer between parallel streams," J. Fluid Mech. 123, 91 (1982).

${ }^{3}$ R. T. Pierrehumbert and S. E. Widnall, "The two- and three-dimensional instability of a spatially periodic shear layer," J. Fluid Mech. 114, 59 (1982).

${ }^{4}$ S. J. Lin and G. M. Corcos, "The mixing layer: Deterministic models of a turbulent flow. Part 3. The effect of plane strain on the dynamics of streamwise vortices," J. Fluid Mech. 141, 139 (1984).

${ }^{5}$ J. S. Lasheras and H. Choi, "Three-dimensional instability of a plane free shear layer: An experimental study of the formation and evolution of streamwise vortices," J. Fluid Mech. 189, 53 (1988).

${ }^{6} \mathrm{~K}$. J. Nygaard and A. Glezer, "Core instability of the spanwise vortices in a plane mixing layer," Phys. Fluids A 2, 461 (1990).

${ }^{7}$ A. K. Prasad and R. J. Adrian, "'Stereoscopic particle image velocimetry applied to liquid flows," Exp. Fluids 15, 49 (1993).

${ }^{8}$ S. M. Soloff, R. J. Adrian, and Z. C. Liu, "Distortion compensation for generalized stereoscopic particle image velocimetry,"'Meas. Sci. Technol. 8, 1441 (1997)

${ }^{9}$ S. S. Collis, S. K. Lele, R. D. Moser, and M. M. Rogers, "The evolution of a plane mixing layer with spanwise nonuniform forcing," Phys. Fluids 6, 381 (1994). 